\title{
Memory Functions of the Additive Markov Chains: Applications to Complex Dynamic Systems
}

\author{
S. S. Melnyk, O. V. Usatenko, and V. A. Yampol'skii * \\ A. Ya. Usikov Institute for Radiophysics and Electronics \\ Ukrainian Academy of Science, 12 Proskura Street, 61085 Kharkov, Ukraine

\begin{abstract}
A new approach to describing correlation properties of complex dynamic systems with long-range memory based on a concept of additive Markov chains (Phys. Rev. E 68, $061107(2003))$ is developed. An equation connecting the memory and correlation function of the system under study is presented. This equation allows reconstructing a memory function using a correlation function of the system. Effectiveness and robustness of the proposed method is demonstrated by simple model examples. Memory functions of concrete coarse-grained literary texts are found and their universal power-law behavior at long distances is revealed.

PACS numbers: 05.40.-a, 02.50.Ga, 87.10.+e
\end{abstract}

The problem of long-range correlated dynamic systems (LRCS) has been under study for a long time in many areas of contemporary physics [1, 2], biology [3, 4], economics 4, 5], etc. 44, 6]. An important example of complex LRCS are naturally written texts [7, 8, 9]. The efficient method for investigating long-range correlations in such systems consists in the decomposition of the space of states into a finite number of parts labelled by definite symbols, which are naturally ordered according to the dynamics of the system. The most frequently used method of the decomposition is based on the introduction of two parts of the phase space. In other words, the approach assumes mapping two kinds of states onto two symbols, say 0 and 1 . Thus, the problem is reduced to investigating the statistical properties of binary sequences.

It might be thought that the coarse graining could result in losing, at least, the shortrange memory in the sequence. The authors of Ref. [10, 11] considered the Markov sequences with a many-valued alphabet. They demonstrated that the mapping of a given sequence

\footnotetext{
* E-mail: yam@ire.kharkov.ua
} 
into a small-alphabet sequence does not necessarily imply that the long-range correlations presented in the initial text would be preserved. Moreover, in general, the coarse-graining procedure could lead to spurious long-range correlations. However, as was shown in Ref. [9], coarse-graining does not destroy the existing correlations in many real symbolic systems. The statistical properties of coarse-grained texts depend, but not significantly, on the kind of mapping. This implies that only a small part of all possible kinds of mapping can slightly change the initial correlations in the system. So, there is no point in coding every symbol (associating every part of the phase space of the system with its binary code) to analyze the correlation properties of the texts, as it is done, for example, in Ref. [8], but it is sufficient to use the coarse-graining procedure.

One of the ways to get a correct insight into the nature of correlations in a system consists in an ability of constructing a mathematical object (for example, a correlated sequence of symbols) possessing the same statistical properties as the initial system. There exist many algorithms for generating long-range correlated sequences: the inverse Fourier transformation 6], the expansion-modification Li method [12], the Voss procedure of consequent random additions [13], the correlated Levy walks [14], etc. 6, 15]. We believe that, among the above-mentioned methods, using the many-step Markov chains is one of the most important, because it offers a possibility to construct a random sequence with definite correlation properties in the most natural way. This was demonstrated in Ref. 16], where the concept of additive Markov chain with the step-like memory function (which allows the analytical treatment) was introduced. There exist some dynamic systems (coarse-grained sequences of Eukarya's DNA and dictionaries) with the correlation properties that can be well described by this model. The concept of additivity, primarily introduced in paper [9], was later generalized for the case of binary non-stationary Markov chains [17]. Another generalization was based on consideration of Markov sequences with a many-valued alphabet [10, 11].

In the present work, we continue investigating into additive Markov chains with more complex memory functions. An equation connecting mutually-complementary characteristics of a random sequence, i.e. the memory and correlation functions, is obtained. Upon finding the memory function of the original random sequence on the basis of the analysis of its statistical properties, namely, its correlation function, we can build the corresponding Markov chain, which possesses the same statistical properties as the initial sequence. Effectiveness and robustness of the proposed method is demonstrated by simple model ex- 
amples. This method is most essential for some applications, e.g., for the construction of correlated sequence of elements which can be used to fabricate the effective filters of electrical or optical signals, Ref. [18]. The suggested method allowed us to find memory functions of concrete coarse-grained literary texts and to reveal their universal power-law behavior at long distances.

Let us consider a homogeneous binary sequence of symbols, $a_{i}=\{0,1\}$. To determine the $N$-step Markov chain we have to introduce the conditional probability $P\left(a_{i} \mid\right.$ $\left.a_{i-N}, a_{i-N+1}, \ldots, a_{i-1}\right)$ of occurring the definite symbol $a_{i}$ (for example, $a_{i}=1$ ) after $N$ word $T_{N, i}$, where $T_{N, i}$ stands for the sequence of symbols $a_{i-N}, a_{i-N+1}, \ldots, a_{i-1}$. Thus, it is necessary to define $2^{N}$ values of the $P$-function corresponding to each possible configuration of the symbols in the $N$-word $a_{i-N}, a_{i-N+1}, \ldots, a_{i-1}$. The value of $N$ is referred to as the memory length of Markov chain.

Considering that we are going to deal with the sequences possessing the memory length of order of $10^{6}$, we need to make some simplification of the $P$-function. We suppose that it has the additive form,

$$
P\left(a_{i}=1 \mid T_{N, i}\right)=\sum_{k=1}^{N} f\left(a_{i-k}, k\right),
$$

and corresponds to the additive influence of the previous symbols upon the generated one. The value of $f\left(a_{i-k}, k\right)$ is the contribution of symbol $a_{i-k}$ to the conditional probability of occurring the symbol unity at the $i$ th site. The homogeneity of the Markov chain is provided by the $i$-independence of conditional probability Eq. (11).

Let us rewrite Eq. (10) in an equivalent form,

$$
P\left(a_{i}=1 \mid T_{N, i}\right)=b+\sum_{r=1}^{N} F(r)\left(a_{i-r}-b\right),
$$

with

$$
b=\frac{\sum_{r=1}^{N} f(0, r)}{1-\sum_{r=1}^{N} F(r)}, \quad F(r)=f(1, r)-f(0, r) .
$$

The constant $b$ is the value of $a_{i}$ averaged over the whole sequence, $b=\bar{a}$ :

$$
\bar{a}=\lim _{M \rightarrow \infty} \frac{1}{2 M+1} \sum_{i=-M}^{M} a_{i} .
$$

Indeed, according to the ergodicity of the Markov chain, $\bar{a}$ coincides with the value of $a_{i}$ 
averaged over the ensemble of realizations of the Markov chain. So, we can write

$$
\bar{a}=\operatorname{Pr}\left(a_{i}=1\right)=\sum_{T_{N, i}} P\left(a_{i}=1 \mid T_{N, i}\right) \operatorname{Pr}\left(T_{N, i}\right)
$$

Here $\operatorname{Pr}\left(a_{i}=1\right)$ is the probability of occurring the symbol $a_{i}$ equal to unity and $\operatorname{Pr}\left(T_{N, i}\right)$ is the probability of occurring the definite word $T_{N, i}$ in the considering ensemble of sequences. Substituting $P\left(a_{i}=1 \mid T_{N, i}\right)$ from Eq. (2) into Eq. (15) and taking into account the obvious relation $\sum_{T_{N, i}} \operatorname{Pr}\left(T_{N, i}\right)=1$, one gets,

$$
\bar{a}=b-b \sum_{r=1}^{N} F(r)+\sum_{r=1}^{N} F(r) \sum_{T_{N, i}} \operatorname{Pr}\left(T_{N, i}\right) a_{i-r}
$$

The sum $\sum_{T_{N, i}} \operatorname{Pr}\left(T_{N, i}\right) a_{i-r}$ does not depend on the subscript $r$ and obviously coincides with $\bar{a}$. So, we have $\bar{a}=b+(\bar{a}-b) \sum_{r} F(r)$. From this equation we conclude that $b=\bar{a}$. Thus, we can rewrite Eq. (2) as

$$
P\left(a_{i}=1 \mid T_{N, i}\right)=\bar{a}+\sum_{r=1}^{N} F(r)\left(a_{i-r}-\bar{a}\right) .
$$

We refer to $F(r)$ as the memory function (MF). It describes the strength of influence of previous symbol $a_{i-r}$ upon a generated one, $a_{i}$. To the best of our knowledge, the concept of memory function for many-step Markov chains was introduced in Ref. [9]. The function $P(. \mid$.) contains the complete information about correlation properties of the Markov chain. Typically, the correlation function and other moments are employed as the input characteristics for the description of the correlated random sequences. However, the correlation function describes not only the direct interconnection of the elements $a_{i}$ and $a_{i+r}$, but also takes into account their indirect interaction via all other intermediate elements. Our approach operates with the "origin" characteristics of the system, specifically, with the memory function. The correlation and memory functions are mutual-complementary characteristics of a random sequence in the following sense. The numerical analysis of a given random sequence enables one to directly determine the correlation function rather than the memory function. On the other hand, it is possible to construct a random sequence using the memory function, but not the correlation one. Therefore, we believe that the investigation of memory function of the correlated systems will permit one to disclose their intrinsic properties which provide the correlations between the elements. 
A dichotomic symbols in a Markov chain can be thought of as the sequence of states of some particle, which participates in a correlated Brownian motion. Every element of the sequence corresponds to the instant change of particle's coordinate. Every $L$-word (the sub-sequence of symbols of the length $L$ in the sequence) can be regarded as one of the realizations of the ensemble of correlated Brownian trajectories in the "temporal" interval $L$. This point of view on the symbolic sequence makes it possible to use the statistical methods for investigating the dynamic systems.

We consider the distribution $W_{L}(k)$ of the words of definite length $L$ by the number $k$ of unities in them, $k_{i}(L)=\sum_{l=1}^{L} a_{i+l}$, and the variance $D(L)$ of $k_{i}(L)$,

$$
D(L)=<(k-<k>)^{2}>,
$$

where the definition of average value of $g(k)$ is $\left\langle g(k)>=\sum_{k=0}^{L} g(k) W_{L}(k)\right.$. It follows from Eq. (7) that the positive MF values result in the persistent diffusion where previous displacements of the Brownian particle in some direction provoke its consequent displacement in the same direction. The negative values of the MF correspond to the anti-persistent diffusion where the changes in the direction of motion are more probable. In terms of the Ising model with long-range particles interactions that could be naturally associated with the Markov chains, the positive (negative) values of the MF correspond to the ferromagnetic (antiferromagnetic) interaction of particles. The additive form (II) of the conditional probability function corresponds to the pair interaction and disregard of many-particles interactions.

The memory function used in Refs. 9, 16] was characterized by the step-like behavior and defined by two parameters only: the memory depth $N$ and the strength of symbol's correlations. Such a memory function describes only one type of correlations in a given system, the persistent or anti-persistent one, which results in the super- or sub-linear dependence $D(L)$ 19]. Obviously, both types of correlations can be observed at different scales in the same system. Thus, one needs to use more complex memory functions for detailed description of the systems with both type of correlations. Besides, we have to find out a relation connecting the mutually-complementary characteristics of random sequence, the memory and correlation functions.

We suggest below two methods for finding the memory function $F(r)$ of a random binary sequence with a known correlation function. The first one is based on the minimization of a "distance" Dist between the Markov chain generated by means of a sought-for MF and the 
initial sequence of symbols. This distance is determined by the formula,

$$
\text { Dist }=\overline{\left(a_{i}-P\left(a_{i}=1 \mid T_{N, i}\right)\right)^{2}}=\lim _{M \rightarrow \infty} \frac{1}{2 M+1} \sum_{i=-M}^{M}\left(a_{i}-P\left(a_{i}=1 \mid T_{N, i}\right)\right)^{2},
$$

with the conditional probability $P$ defined by Eq. (77).

Let us express distance (9) in terms of the correlation function,

$$
K(r)=\overline{a_{i} a_{i+r}}-\bar{a}^{2}, \quad K(0)=\bar{a}(1-\bar{a}), \quad K(-r)=K(r) .
$$

From Eqs. (7), (9), one obtains

$$
\begin{gathered}
\text { Dist }=\sum_{r, r^{\prime}} \overline{\left(a_{i-r}-\bar{a}\right)\left(a_{i-r^{\prime}}-\bar{a}\right)} F(r) F\left(r^{\prime}\right)-2 \sum_{r} \overline{\left(a_{i}-\bar{a}\right)\left(a_{i-r}-\bar{a}\right)} F(r)+\overline{\left(a_{i}-\bar{a}\right)^{2}} \\
=\sum_{r, r^{\prime}} K\left(r-r^{\prime}\right) F(r) F\left(r^{\prime}\right)-2 \sum_{r} K(r) F(r)+K(0) .
\end{gathered}
$$

The minimization equation,

$$
\frac{\delta \text { Dist }}{\delta F(r)}=2 \sum_{r^{\prime}} K\left(r-r^{\prime}\right) F\left(r^{\prime}\right)-2 K(r)=0,
$$

yields the relationship between the correlation and memory functions,

$$
K(r)=\sum_{r^{\prime}=1}^{N} F\left(r^{\prime}\right) K\left(r-r^{\prime}\right), \quad r \geq 1 .
$$

Equation (13) can also be derived by straightforward calculation of the average $\overline{a_{i} a_{i+r}}$ in Eq. (10) using definition (17) of the memory function.

The second method resulting from the first one, establishes a relationship between the memory function $F(r)$ and the variance $D(L)$,

$$
\begin{gathered}
M(r, 0)=\sum_{r^{\prime}=1}^{N} F\left(r^{\prime}\right) M\left(r, r^{\prime}\right), \\
M\left(r, r^{\prime}\right)=D\left(r-r^{\prime}\right)-\left(D\left(-r^{\prime}\right)+r\left[D\left(-r^{\prime}+1\right)-D\left(-r^{\prime}\right)\right]\right) .
\end{gathered}
$$

It is a set of linear equations for $F(r)$ with coefficients $M\left(r, r^{\prime}\right)$ determined by $D(r)$. The relations, $K(r)=[D(r-1)-2 D(r)+D(r+1)] / 2$ obtained in Ref. [9] and $D(-r)=D(r)$ are used here.

Let us verify the robustness of our method by numerical simulations. We consider a model "triangle" memory function,

$$
F(r)=0.008 \begin{cases}r, & 1 \leq r<10 \\ 20-r, & 10 \leq r<20 \\ 0, & r \geq 20\end{cases}
$$




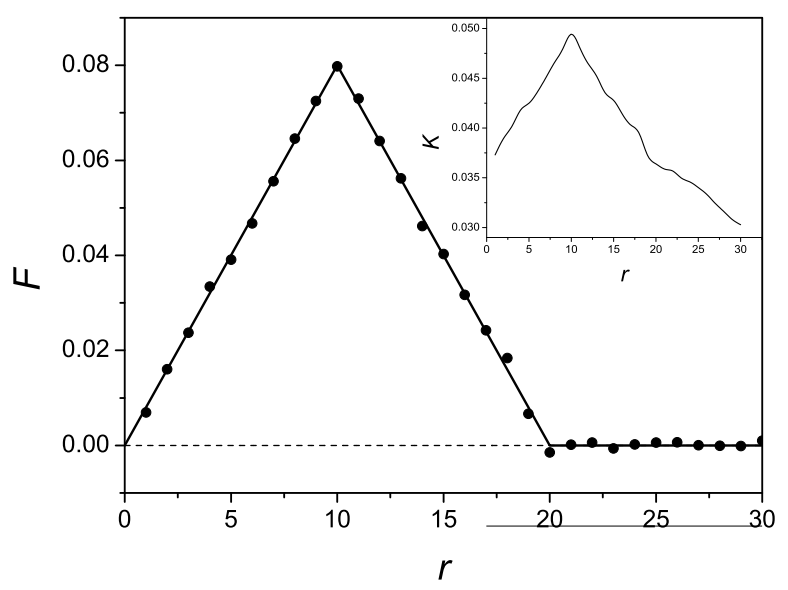

FIG. 1: The initial memory function Eq. (15) (solid line) and the reconstructed one (dots) vs the distance $r$. In inset, the correlation function $K(r)$ obtained by a numerical analysis of the sequence constructed by means of the memory function Eq. (15).

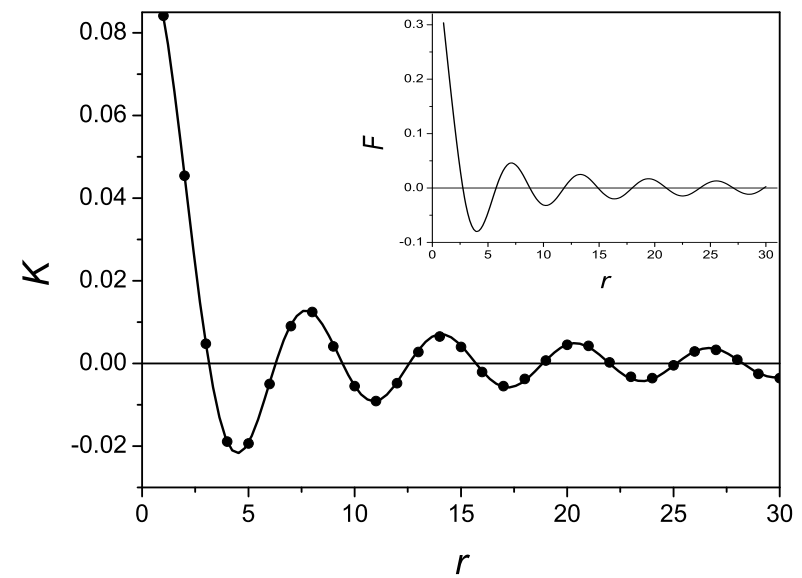

FIG. 2: The model correlation function $K(r)$ described by Eq. (16) (solid line). The dots correspond to the reconstructed correlation function. In inset, the memory function $F(r)$ obtained by numerical solution of Eq. (13) with correlation function Eq. (16).

presented in Fig. 11 by solid line. Using Eq. (17), we construct a random non-biased, $\bar{a}=1 / 2$, sequence of symbols $\{0,1\}$. Then, with the aid of the constructed binary sequence of the length $10^{6}$, we calculate numerically the correlation function $K(r)$. The result of these calculations is presented in inset Fig. 1. One can see that the correlation function $K(r)$ mimics roughly the memory function $F(r)$ over the region $1 \leq r \leq 20$. In the region $r>20$, the memory function is equal to zero but the correlation function does not vanish [20]. Then, using the obtained correlation function $K(r)$, we solve numerically Eq. (13). The result is 
shown in Fig. 1 by dots. One can see a good agrement of initial, Eq. (15), and reconstructed memory functions $F(r)$.

The main and very nontrivial result of our paper consists in the ability to construct a binary sequence with an arbitrary prescribed correlation function by means of Eq. (13). As an example, let us consider the model correlation function,

$$
K(r)=0.1 \frac{\sin (r)}{r}
$$

presented by the solid line in Fig. 2. We solve Eq. (13) numerically to find the memory function $F(r)$ using this correlation function. The result is presented in inset Fig. 2, Then we construct the binary Markov chain using the obtained memory function $F(r)$. To check up a robustness of the method, we calculate the correlation function $K(r)$ of the constructed chain (the dots in Fig. 21) and compare it with Eq. (16). One can see an excellent agreement between the initial and reconstructed correlation functions.

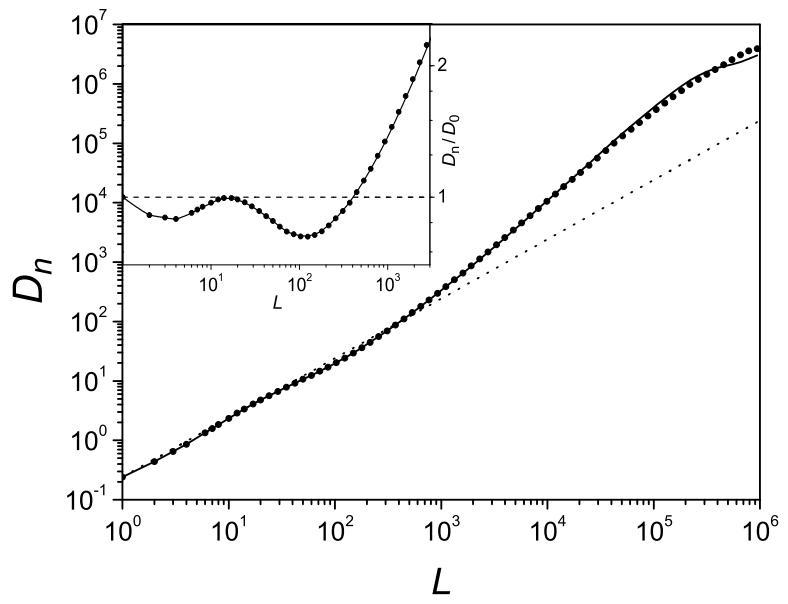

FIG. 3: The normalized variance $D_{n}(L)$ for the coarse-grained text of Bible (solid line) and for the sequence generated by means of the reconstructed memory function $F(r)$ (dots). The dotted straight line describes the non-biased non-correlated Brownian diffusion, $D_{0}(L)=L / 4$. The inset demonstrates the anti-persistent dependence of ratio $D_{n}(L) / D_{0}(L)$ on $L$ at short distances.

Let us demonstrate the effectiveness of our concept of the additive Markov chains when investigating the correlation properties of coarse grained literary texts. First, we use the coarse-graining procedure and map the letters of the text of Bible [21] onto the symbols zero and unity (here, $(a-m) \mapsto 0,(n-z) \mapsto 1)$. Then we examine the correlation properties of the constructed sequence and calculate numerically the variance $D(L)$. The result of 
simulation of the normalized variance $D_{n}(L)=D(L) / 4 \bar{a}(1-\bar{a})$ is presented by the solid line in Fig. 3. The dominator $4 \bar{a}(1-\bar{a})$ in the equation for the normalized variance $D_{n}(L)$ is inserted in order to take into account the inequality of the numbers of zeros and unities in the coarse-grained literary texts. The straight dotted line in this figure describes the variance $D_{0}(L)=L / 4$, which corresponds to the non-biased non-correlated Brownian diffusion. The deviation of the solid line from the dotted one demonstrates the existence of correlations in the text. It is clearly seen that the diffusion is anti-persistent at small distances, $L \lesssim 300$, (see inset Fig. (3) whereas it is persistent at long distances.

The memory function $F(r)$ for the coarse-grained text of Bible at $r<300$ obtained by numerical solution of Eq. (14) is shown in Fig. (4) At long distances, $r>300$, the memory function can be nicely approximated by the power function $F(r)=0.25 r^{-1.1}$, which is presented by the dash-dotted line in inset Fig. 目

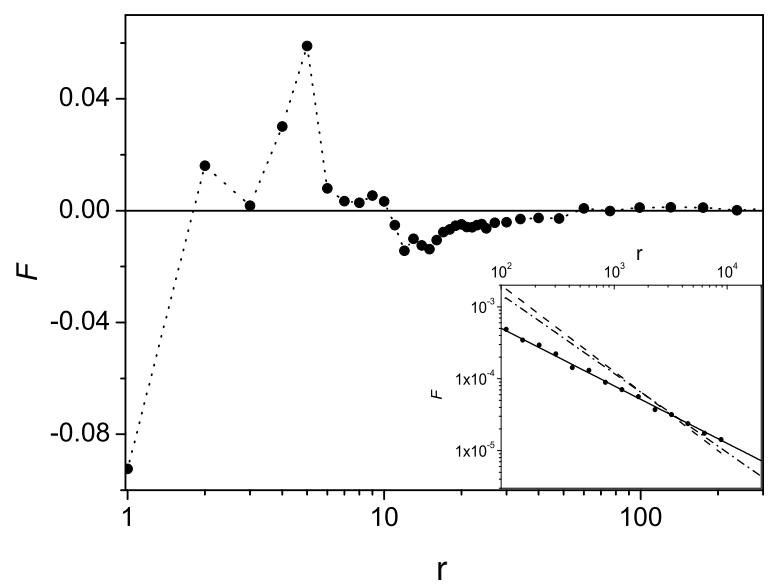

FIG. 4: The memory function $F(r)$ for the coarse-grained text of Bible at short distances. In inset, the power-law decreasing portions of the $F(r)$ plots for several texts. The dots correspond to "Pygmalion" by B. Shaw. The solid line corresponds to power-law fitting of this function. The dash dotted and dashed lines correspond to Bible in English and Russian, respectively.

Note that the region $r \lesssim 40$ of negative anti-persistent memory function provides much longer distances $L \sim 300$ of anti-persistent behavior of the variance $D(L)$.

Our study reveals the existence of two characteristic regions with different behavior of the memory function and, correspondingly, of persistent and anti-persistent portions in the $D(L)$ dependence. This appears to be a prominent feature of all texts written in any language.

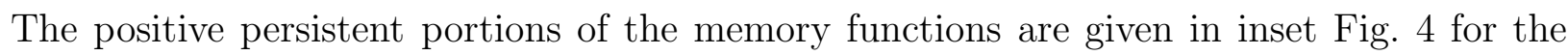


coarse-grained English- and Russian-worded texts of Bible (dash-dotted and dashed lines, Refs. [21] and [22], correspondingly). Besides, for comparison, the memory function of the coarse-grained text of "Pygmalion" by B. Shaw [23] is presented in the same inset (dots), the power-law fitting is shown by solid line.

It is interesting to note that the memory function of any text mimics the correlation function, as it was found for the model example Eq. (16). This fact is confirmed by Fig. 5 where the correlation function of the coarse-grained text of Bible is shown. One can see that its behavior at both short and long scales is similar to the memory function presented in Fig. 4. However, the exponents in the power-law approximations of $K(r)$ and $F(r)$ functions differ essentially.

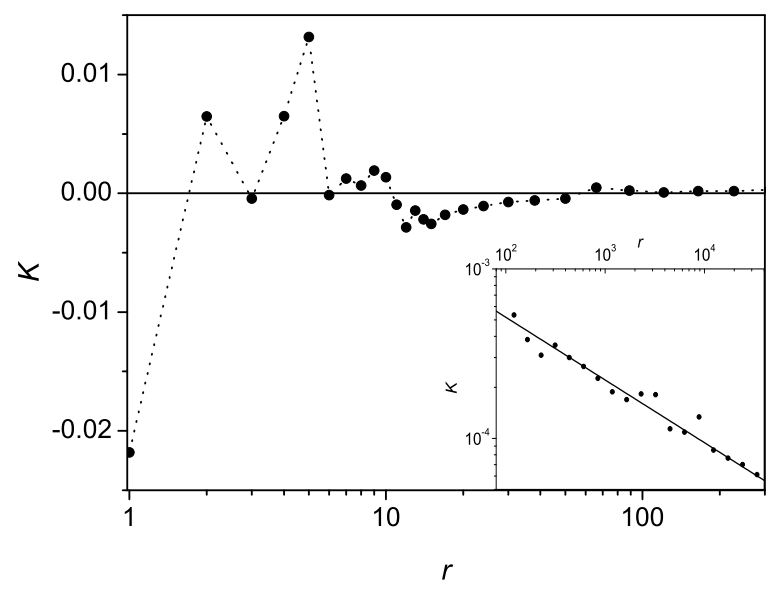

FIG. 5: The correlation function $K(r)$ for the coarse-grained text of Bible at short distances. In inset, the power-law decreasing portions of the $K(r)$ plot for the same text. The solid line corresponds to power-law fitting of this function.

Thus, we have demonstrated the efficiency of description of the symbolic sequences with long-range correlations in terms of the memory function. An equation connecting the memory and correlation functions of the system under study is obtained. This equation allows reconstructing a memory function using a correlation function of the system. Actually, the memory function appears to be a suitable informative "visiting card" of any symbolic stochastic process. The effectiveness and robustness of the proposed method is demonstrated by simple model examples. Memory functions for some concrete examples of the coarse-grained literary texts are constructed and their power-law behavior at long distances is revealed. Thus, we have shown the complexity of organization of the literary texts in 
contrast to a previously discussed simple power-law decrease of correlations [24].

Our theory describes not all statistical properties of the binary symbolic sequences. For example, our consideration cannot reflect the property of directivity of the texts since the theory is based on the examination of the correlation function that is even by definition. This property can be revealed only using the ternary (or of the higher order) correlation functions. The linguistic aspects of the problem also require a regular and systematic study.

We have examined the simplest examples or random sequences, the dichotomic one. Nevertheless, our preliminary consideration suggests that the presented theory can by generalized to the arbitrary additive Markov process with a finite or infinite number of states and with discrete or continuous "time". A study in this direction is in progress.

The proposed approach can be used for the analysis of other correlated systems in different fields of science.

[1] U. Balucani, M. H. Lee, V. Tognetti, Phys. Rep. 373, 409 (2003).

[2] I. M. Sokolov, Phys. Rev. Lett. 90, 080601 (2003).

[3] R. F. Voss, Phys. Rev. Lett. 68, 3805 (1992).

[4] H. E. Stanley et. al., Physica A 224,302 (1996).

[5] R. N. Mantegna, H. E. Stanley, Nature (London) 376, 46 (1995).

[6] A. Czirok, R. N. Mantegna, S. Havlin, and H. E. Stanley, Phys. Rev. E 52, 446 (1995).

[7] A. Schenkel, J. Zhang, and Y. C. Zhang, Fractals 1, 47 (1993).

[8] P. Kokol, V. Podgorelec, Complexity International, 7, 1 (2000).

[9] O. V. Usatenko, V. A. Yampol'skii, S. S. Mel'nyk, and K. E. Kechedzhy, Phys. Rev. E 68, 061107 (2003).

[10] S. L. Narasimhan, J. A. Nathan, and K. P. N. Murthy, Europhys. Lett. 69 (1), 22 (2005).

[11] S. L. Narasimhan, J. A. Nathan, P. S. R. Krishna, and K. P. N. Murthy, arXiv:cond-mat/0409053.

[12] W. Li, Europhys. Let. 10, 395 (1989).

[13] R. F. Voss, in: Fundamental Algorithms in Computer Graphics, ed. R. A. Earnshaw (Springer, Berlin, 1985) p. 805.

[14] M. F. Shlesinger, G. M. Zaslavsky, and J. Klafter, Nature (London) 363, 31 (1993). 
[15] I. F. Herbut, arXiv:cond-mat/0007266.

[16] O. V. Usatenko and V. A. Yampol'skii, Phys. Rev. Lett. 90, 110601 (2003).

[17] S. Hod and U. Keshet, Phys. Rev. E 70, 015104(R) (2004).

[18] F. M. Izrailev, A. A. Krokhin, and S. E. Ulloa, Phys. Rev. B 63, 041102(R) (2001).

[19] Note that we here discuss the dependence of the variance $D$ upon the length $L$ that describes the persistent (or antipersistent) correlations in the words of different lengths $L$. This length does not coincides with the memory length $N, L \neq N$. The dependence $D(L)$ is completely different from the dependence $D$ on the memory length $N$ discussed in Ref. [10, 11].

[20] The existence of the "additional tail" in the correlation function is in agreement with Ref. [9] and corresponds to the well known fact that the correlation length is always larger then the region of memory function action.

[21] The Old Testament of the King James Version of the Bible, http://www.writersbbs.com/bible/

[22] Russian Synodal LiO 31/7/91, http: //lib.ru/ hristian/bibliya/nowyj_zawet.txt.

[23] http://eserver.org/drama/pygmalion/default.html.

[24] I. Kanter, D. A. Kessler, Phys. Rev. Lett. 74, 22 (1995). 